\title{
Accompagner le personnel enseignant dans les choix d'utilisation d'une ressource technopédagogique : Un questionnement technopédagogique utile à l'intégration des technologies en enseignement dans les programmes d'études au collégial
}

Christine Gaucher, Claude Martel et Léane Arsenault

\section{(2) OpenEdition}

Journals

Édition électronique

URL : http://journals.openedition.org/trema/3369

DOI : $10.4000 /$ trema.3369

ISSN : 2107-0997

Éditeur

Faculté d'Éducation de l'université de Montpellier

Édition imprimée

Date de publication : 1 mai 2016

Pagination : 25-35

ISBN : 979-10-96627-00-4

ISSN : 1167-315X

Référence électronique

Christine Gaucher, Claude Martel et Léane Arsenault, « Accompagner le personnel enseignant dans les choix d'utilisation d'une ressource technopédagogique : Un questionnement technopédagogique utile à l'intégration des technologies en enseignement dans les programmes d'études au collégial », Tréma [En ligne], 44 | 2016, mis en ligne le 01 juin 2016, consulté le 30 avril 2019. URL : http:// journals.openedition.org/trema/3369; DOI : 10.4000/trema.3369

Ce document a été généré automatiquement le 30 avril 2019

Trema 


\section{Accompagner le personnel enseignant dans les choix d'utilisation d'une ressource technopédagogique : Un questionnement technopédagogique utile à l'intégration des technologies en enseignement dans les programmes d'études au collégial}

Christine Gaucher, Claude Martel et Léane Arsenault

Note: Dans le contexte du présent article, nous avions un souci d'applicabilité. Cet article a donc été rédigé selon les étapes apparentées à une recherche-développement en respectant le devis méthodologique tel que proposé par Loiselle et Harvey $(2007 ; 2009)$. Nous nous inscrivons ainsi en cohérence avec Simmoneaux (2011 p. 3) qui affirme : la recherche scientifique n'est pas neutre; elle est porteuse de valeurs: "universalité, désintérêt, scepticisme engagé, simplicité, prédictibilité, applicabilité».

\section{Idée de développement}

Historiquement, l'intégration de l'ordinateur en classe se voulait fort prometteuse. L'ordinateur était promis à une augmentation de la " productivité » tant de l'enseignant que de l'étudiant. Enseigner et apprendre davantage en moins de temps, clamaient les experts! Cependant, en plus d'une difficile accessibilité aux équipements, les études démontraient qu'ils avaient peu d'impact sur le plan pédagogique et remettaient une 
bonne partie du blâme aux enseignants. Pendant plusieurs années, il était fréquent d'observer une séquence "Excitation - désillusion - blâme » (Cuban, 1993) à l'arrivée de technologies toute aussi prometteuses les unes que les autres. Il n'est alors pas étonnant que les enseignants tardent à les utiliser et y soient même parfois réfractaires.

De plus, il est important de rappeler que les premières activités de formation à distance ont été créées pour répondre à des contraintes d'ordre géographique ou temporel (Deschenes et Maltais, 2006), bref une mesure d'accessibilité, un palliatif à défaut de la présence plutôt qu'y voir des occasions technopédagogiques de faciliter l'apprentissage. Ajoutons la perception d'une complexité technique qui place les technologies numériques comme une difficulté et non pas comme une ressource (Moroney et Haigh, 2011). Or, il devient impératif de dépasser ce stade au collégial, entre autres en raison d'un profil TIC que l'on retrouve dans plusieurs programmes d'études au collégial. Un développement minimal de la compétence est devenu un incontournable pour le corps enseignant, voire une obligation (Barrette, 2005)! En réponse, dès la conception de programmes de deuxième cycle en $2005^{1}$, le secteur PERFORMA de l'Université de Sherbrooke, dédié à la formation et au perfectionnement des maîtres de l'enseignement au collégial, a intégré dans le profil de sortie de la Maîtrise en enseignement au collégial (MEC) un ensemble de compétences dont l'intégration efficace de technologies dans l'enseignement et l'apprentissage.

Barrette (Ibid.) étudie l'objet en posant la question : «Qu'est-ce que les professeurs font mieux en exploitant efficacement les technologies de l'information et de la communication?». Pour y répondre, il propose une typologie des différents domaines dans lesquels les enseignants utilisent les technologies numériques pour améliorer l'efficacité et la portée de leurs actions professionnelles. L'ensemble des domaines sont reliés par un dénominateur commun: la maîtrise initiale de concepts et d'habiletés de base dans le domaine des TIC. Barrette (2005) ajoute que si l'ensemble des programmes de formation au collégial (enseignement supérieur) vise le développement minimal d'une maîtrise fonctionnelle des systèmes ordinés par les finissants, l'acquisition de cette maîtrise devrait donc être assurée par tous les enseignants de l'ordre collégial. Plus récemment, deux autres chercheurs intéressés à la formation des enseignantes et enseignants du collégial, Poellhuber et Fournier-St-Laurent (2014), précisent qu'il s'agit certes «d'une condition nécessaire, mais pas suffisante. En réalité, la composante pédagogique doit se jumeler à cette compétence technique ce qui nous amène à parler de compétences technopédagogiques » (p. 162).

En 2013, une équipe de conseillers en intégration des technologies a expérimenté un outil de réflexion issu du cadre de référence de Mishra et Koehler (2006). Cette expérimentation visait à vérifier l'efficacité de ce dernier pour identifier des ressources technopédagogiques en lien avec la professionnalisation de l'enseignant.

\section{Référentiel}

6 À la recherche d'un cadre conceptuel qui permettrait d'établir des liens entre l'utilisation pédagogique des technologies et d'autres considérations comme le rapport aux savoirs ou encore la situation professionnelle en cause, notre choix s'est arrêté sur un premier cadre de référence qui est le TPaCK (Ibid.). On peut traduire l'acronyme TPaCK (Technological Pedagogical Content Knowledge) comme la compétence à intégrer les technologies dans des activités d'enseignement et d'apprentissage liées à sa discipline. La compétence TPaCK 
correspond à l'intersection entre les trois domaines de connaissances dites primaires du personnel enseignant, respectivement les connaissances disciplinaires (Content Knowledge), pédagogiques (Pedagogical Knowledge) et technologiques (Technological Knowledge).

Une traduction libre de la définition de ces connaissances se lit comme suit :

Figure 1: Le modèle TPaCK (Mishra et Koehler, 2006).

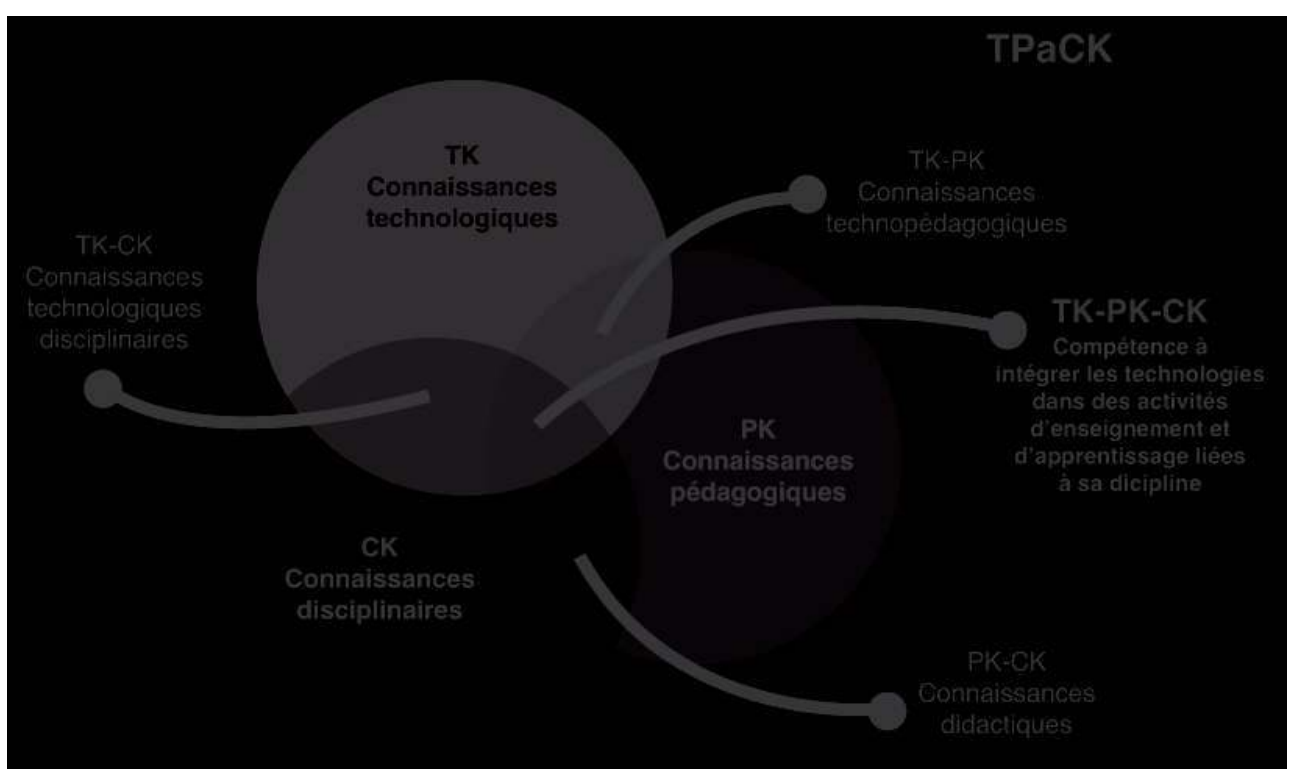

\section{Connaissances disciplinaires}

Les connaissances disciplinaires font généralement référence à l'expertise de l'enseignant. Outre l'étendue des connaissances dans la discipline, il faut considérer la pertinence et la sélection des contenus essentiels à enseigner. Ajoutons que, selon Lapierre (2008), cette connaissance disciplinaire est une assise de la construction d'une identité professorale distincte. En effet, elle réfère à la représentation que l'enseignant se fait de sa discipline ou de sa spécialité, à la compréhension qu'il a du programme d'études et de son contenu et au lien qu'il établit entre sa discipline, son contenu et les connaissances des étudiants.

\section{Connaissances pédagogiques}

Raby et Viola (2007) définissent le courant pédagogique comme «le cadre théorique (paradigme éducationnel) et idéologique (paradigme socioculturel) qui détermine l'orientation générale $\mathrm{du}$ processus enseignement - apprentissage dans un contexte d'apprentissage donné » (p. 20). Depuis le début des années 2000, avec l'avènement des programmes par compétences, l'approche socioconstructiviste est souvent privilégiée parce qu'elle s'intéresse à l'apprentissage plutôt qu'à l'enseignement. Plusieurs méthodes ou stratégies d'enseignement qui intègrent les TIC découlent de cette approche qui mise sur « le rôle des interactions entre le sujet et son environnement dans un processus actif qui lui permet de développer des connaissances sur le monde» (Legendre, 2005, dans 
Raby et Viola, 2007, p. 20). Les connaissances pédagogiques incluent donc l'expertise pédagogique, le savoir-agir en situation d'enseignement, mais aussi la pratique réflexive de l'acte d'enseigner un savoir disciplinaire. Plusieurs cadres théoriques peuvent servir pour le questionnement pédagogique. Ici, nous situons en lien avec la discipline d'enseignement le courant pédagogique préconisé et auquel l'enseignant adhère (socioconstructivisme, cognitivisme, behaviorisme, etc.). Quelle est la stratégie d'enseignement et d'évaluation privilégiée... ou comment enseigner et évaluer pour que les étudiantes et les étudiants apprennent?

\section{Connaissances technologiques}

Enfin, les connaissances technologiques ont évolué rapidement avec l'avènement des technologies. Dans le contexte de l'enseignement - apprentissage, il s'agit des connaissances qu'ont les enseignants sur la potentialité et les limites des technologies numériques. Ici se pose la question des connaissances dont l'enseignant dispose ou devrait disposer pour évaluer la pertinence d'une technologie dans son enseignement (Harris, 2008, dans Moroney et Haigh, 2011).

\section{Les trois domaines de connaissance en superposition}

11 Il existe quatre autres domaines de connaissances situés aux intersections des connaissances primaires, soit les connaissances superposées disciplinaires et technologiques (CK$T K$ ), disciplinaires et pédagogiques (CK-PK), pédagogiques et technologiques (PK-TK) et enfin disciplinaires, pédagogiques et technologiques (CK-PK-TK). À l'intersection des connaissances disciplinaires et technologiques, l'enseignant doit maintenir ses connaissances tant dans sa discipline que dans l'utilisation professionnelle des technologies dans le contexte de sa discipline. En ce qui a trait à la superposition entre discipline et pédagogie, l'enseignant doit sélectionner les stratégies pédagogiques les plus pertinentes et adaptées à son contenu disciplinaire. En ce qui concerne les connaissances technopédagogiques, situées à l'intersection des connaissances technologiques et pédagogiques, la compétence recherchée est celle de pouvoir intégrer les technologies dans des activités d'enseignement et d'apprentissage liées à sa discipline. L'équilibre professionnel de l'enseignant se situe à l'intersection des trois connaissances primaires du TPaCK (Mishra et Koehler, 2006). La connaissance et l'analyse de chacune de ces variables et de leurs interactions permettent au personnel enseignant d'évaluer et de développer sa pratique pédagogique. C'est ce que nous appelons le questionnement technopédagogique. La production d'un outil de réflexion s'inscrit dans ce contexte d'établir la première base de l'approche réflexive essentielle au développement de la compétence à utiliser des stratégies pédagogiques qui intègrent efficacement les technologies dans l'enseignement des contenus disciplinaires ${ }^{2}$.

$12 \mathrm{Au}$ fil des travaux, il est apparu pertinent de greffer le questionnement technopédagogique à celui issu des travaux sur la didactique (connaissances disciplinaires) du Secteur PERFORMA. Tel que développée par Bizier (2008), la démarche réflexive propose cinq (5) entrées pour un questionnement qui reprend les trois (3) compétences primaires énoncées dans le TPaCK. Le tableau 1 illustre l'exploration de ces 
compétences TPaCK à l'aide du questionnement didactique de Bizier, enrichi de questions relatives à la dimension du matériel pédagogique qui ont été puisées dans les écrits de Poellhuber et Fournier-St-Laurent (2014). L'enseignant peut ainsi créer un équilibre entre les trois domaines de connaissances et établir les liens de cohérence à travers ses choix ou les changements qu'il souhaite apporter à son enseignement.

Tableau 1 : Le questionnement technopédagogique

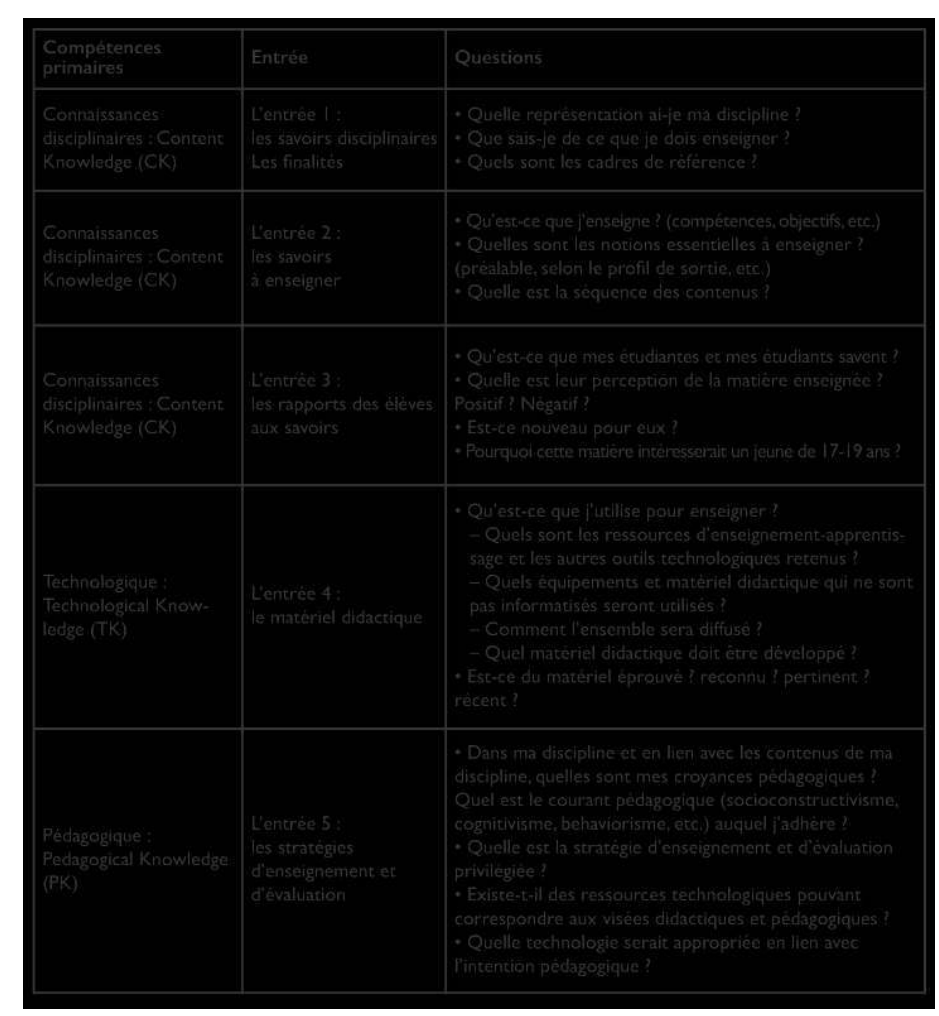

\section{Méthodologie et opérationnalisation}

Selon Loiselle et Harvey (2007), la finalité des mises à l'essai proposées par la plupart des démarches de recherche développement, souvent associées principalement à la validation du produit, peut être considérée selon une autre perspective [...]. Nielsen (2003), introduit le concept d'utilisabilité (usability) où la qualité de l'expérience d'utilisation du produit, la facilité d'utilisation et la satisfaction de l'usager face au produit sont, entre autres, considérées. Dans cette optique, les mises à l'essai peuvent aussi devenir des moyens d'analyser les interactions entre l'objet en cours de développement et les personnes auxquelles s'adresse cet objet (p. 42).

14 Ainsi, nous avons mis à l'essai le référentiel, soit le TPaCK, en prenant soin de concevoir un outil de réflexion rappelant les grandes lignes du référentiel et permettant, dans un premier temps, l'idéation et, par la suite, la production de scénarios pédagogiques tenant compte des trois domaines de connaissances. À la suite de la mise à l'essai, un groupe de discussion nous a permis de recueillir des informations de différentes natures. 


\section{Conception et réalisation d'un outil conceptuel et technique}

15 La collecte de données devait servir à créer un répertoire de ressources technopédagogiques contenant des scénarios inspirants pour les enseignantes et enseignants au collégial. Or une première étape dans la mise en place de l'expérimentation a permis l'élaboration d'un outil de collecte de données sous la forme de fiches réflexives contenant les informations utiles aux participants. Dans la fiche ciaprès, le cadre conceptuel TPaCK y est représenté et une section permettant d'écrire le contenu disciplinaire, (CK), la stratégie pédagogique (PK) la technologie (TK) était à compléter. Dans la partie scénario, un espace était réservé à la description d'une situation d'enseignement/apprentissage déjà vécue ou imaginée se situant à l'intersection des trois connaissances primaires, donc la compétence TPaCK.

Figure 2 : Fiche réflexive TPaCK : Mise à l'essai et validation (Martel, 2013)

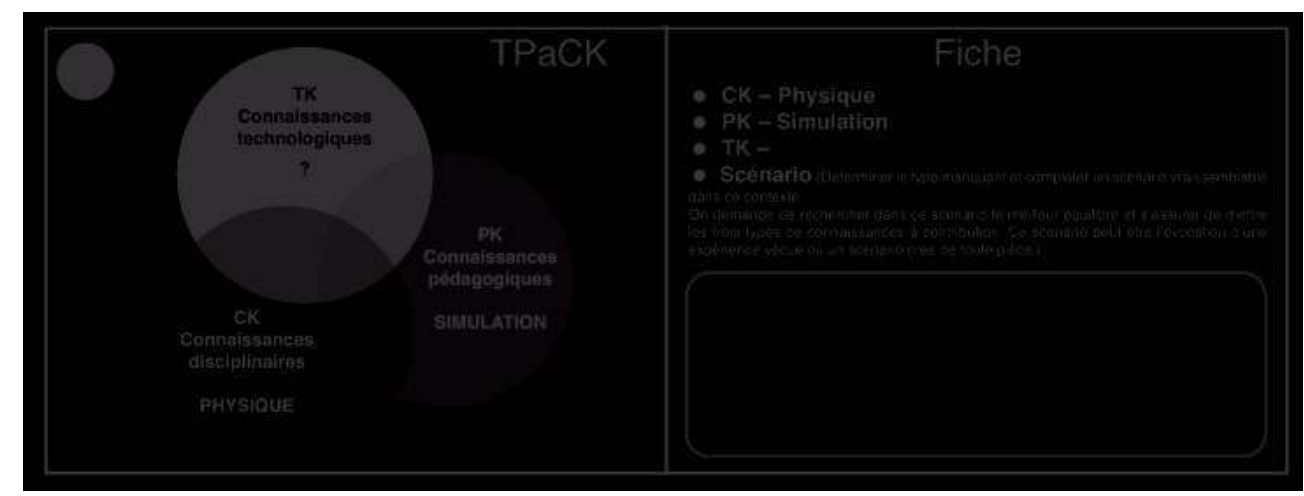

Souhaitant élaborer une première banque de ressources technopédagogiques et vérifier à la fois la pertinence et l'efficacité du questionnement technopédagogique, une activité d'idéation à partir de cette fiche de réflexion TPaCK a été proposée lors de trois ateliers, l'un tenu en juin 2013 et les deux autres en février 2014. La tenue de ces ateliers a permis la participation de 179 enseignants et conseillers pédagogiques de l'ordre collégial.

Lors des trois ateliers, les membres du groupe de travail en intégration des technologies de PERFORMA ont d'abord expliqué les cadres de référence et les définitions, puis ils ont discuté avec les participants afin de valider leur compréhension du cadre de référence.

Des fiches de réflexion TPaCK ont été distribuées. Les participants ont travaillé en équipe de 5 et disposaient de 90 minutes pour écrire des activités enseignement-apprentissage dans lesquelles l'enseignant combine les trois types de connaissances. Ils avaient le choix de répondre sur papier ou directement dans un outil de compilation (Google formulaire). Chaque équipe a soumis quelques scénarios ( 2 à 5 , voir Annexe 1 ) regroupant les trois connaissances primaires. Par la suite, un groupe de discussion a permis de constater que cette façon de rechercher des ressources d'intégration des TIC aux activités d'enseignement et d'apprentissage était inspirante et assez efficace. 


\section{Les participants} conceptuels et techniques, a permis de recueillir les commentaires des participants sur la qualité de l'expérience d'utilisation de l'outil et sa facilité d'utilisation, ainsi que sur la satisfaction des participants face à l'outil proposé.

\section{Qualité de l'expérience d'utilisation de l'outil et sa facilité d'utilisation}

Tel que décrit précédemment, l'appropriation et la mise à l'essai du référentiel TPaCK ont été réalisées de façon concomitante. Les participants auraient toutefois apprécié un temps de réflexion entre les deux étapes afin de permettre un meilleur 
approfondissement des concepts ainsi qu'une élaboration de scénarios plus complets. En effet, tel que mentionné plus haut, la durée de 90 minutes pour comprendre, appliquer les principes du référentiel et tenir le retour en plénière peut sembler insuffisante. Il importe de mentionner que les caractéristiques des participants étant, pour la plupart, des conseillers pédagogiques et des enseignants intéressés à l'intégration des TIC permettaient une forte présomption à l'effet que les concepts relatifs à la connaissance disciplinaire et à la pédagogie étaient déjà assimilés. Il n'en demeure pas moins que la mise en relation des trois domaines de connaissances (PK, CK, TK) demande une réflexion et un approfondissement.

\section{Satisfaction des participants face à l'outil proposé}

Malgré la durée plutôt restreinte de l'activité, les participants ont trouvé l'outil - soit la fiche de réflexion TPaCK - pertinent pour élaborer des scénarios de qualité. Par contre, ils mentionnent que l'idéation utile à l'élaboration de scénarios a surtout été possible grâce au travail en équipe. Ainsi, le contexte de la mise à l'essai qui prévoyait le travail en équipe doit absolument être associé au succès de l'élaboration de plusieurs scénarios.

Les participants constatent que l'utilisation du référentiel TPaCK pour l'intégration des technologies incite à varier davantage les stratégies d'enseignement - apprentissage en classe. Certains scénarios qui prévoient un travail collaboratif supposent un accompagnement de l'enseignant et un engagement de l'étudiant pour les mettre en œuvre.

Quoi qu'il en soit, la maitrise des technologies demeure un enjeu important. Maitriser une nouvelle plateforme ou technologie implique une appropriation de l'enseignant qui en rebute plusieurs par son caractère chronophage.

Les participants soulignent à grands traits l'importance de vérifier les compétences des étudiants concernant l'utilisation d'une plateforme ou d'un logiciel choisi. Afin d'éviter un trop grand nombre d'heures d'appropriation non pondérées dans la charge de l'étudiant, les technologies doivent obligatoirement avoir un caractère accessible et convivial.

Finalement, l'ensemble des commentaires sur la satisfaction des participants face à l'outil proposé soulève des conditions d'applicabilité de l'outil qu'il convient de prendre en compte. En outre, les participants ont même exprimé certains avertissements plus pratiques quant à la production de matériel didactique, notamment l'enregistrement d'exposés théoriques en classe qui supposent l'obtention des autorisations de diffusion de la part de tous les étudiants et des autres personnes présentes. Outre les dites autorisations, ils ont également insisté sur des détails comme la taille des fichiers, leur classement, leur identification, etc.

La mise en mots de ces conditions témoigne du cheminement réflexif parcouru pendant cette mise à l'essai. 


\section{Conclusion} utilisation des technologies à l'aide de cadres de référence liés aux trois domaines de connaissances, nommément les connaissances disciplinaires, pédagogiques et technologiques. théorique de l'intégration des technologies. Il s'agissait d'un exercice qui ne les confrontait pas dans l'immédiat à mettre les scénarios développés en pratique et de prévoir les obstacles d'ordre personnel ou organisationnel.

Il serait intéressant de reprendre l'exercice en équipe disciplinaire ou équipe programme afin d'expérimenter, selon les forces de chacun, une réelle planification d'intégration des technologies dans l'enseignement des membres de l'équipe. D'autres questions de recherche surgissent : la fiche de réflexion TPaCK favorise-t-elle une perception positive des enseignants à l'égard des technologies ? Est-ce que les enseignants seront en mesure de mettre en pratique les scénarios planifiés? Quelques mois plus tard, ces enseignants ou conseillers pédagogiques ont-ils été en mesure de réaliser les scénarios planifiés?

Annexe 1 : Exemples de scénarios élaborés lors des ateliers

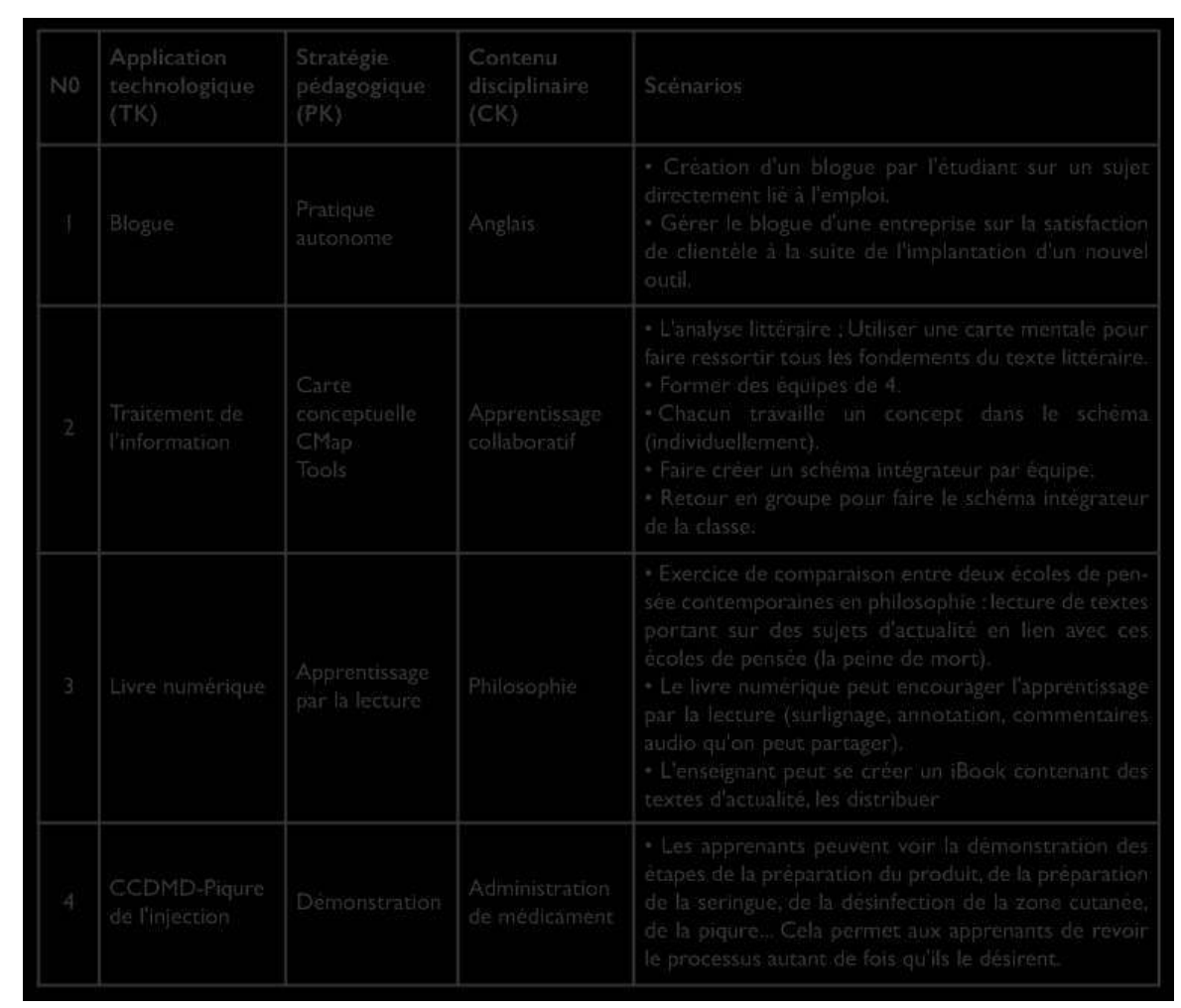




\section{BIBLIOGRAPHIE}

Barrette, C. (2005). Cadre opérationnel des formations PERFORMA visant le développement de la compétence à intégrer les TIC à sa pratique professionnelle. Regroupement des collèges Performa. Sherbrooke : Université de Sherbrooke.

Bizier, N. (2008). Choisir des contenus reconnus et pertinents : un geste professionnel didactique majeur. Pédagogie collégiale, 21(2), 13-18.

Cuban, L. (1993). Computers Meet Classroom: Classroom Wins. Teachers College Record, 95(2), 185-210.

Deschenes, A.-J. et Maltais, M. (2006). Formation à distance et accessibilité. Québec : Les publications Télé-Université.

Mishra, P. et Koehler, M. J. (2006). Technological Pedagogical Content Knowledge: A Framework for Teacher Knowledge. Teachers College Record, 108(6), 1017-1054. Document téléaccessible à l'adresse : http://punya.educ.msu.edu/publications/journal_articles/mishra-koehler-tcr2006.pdf Lapierre, L. (2008). Un cadre de référence pour le questionnement didactique au collégial. Pédagogie collégiale, 21(2), 5-12.

Loiselle, J. et Harvey, S. (2007). La recherche développement en éducation : fondements, apports et limites. Recherches qualitatives, 27(1), 40-59.

Loiselle, J. et Harvey, S. (2009). Proposition d'un modèle de recherche développement. Recherches qualitatives, 28(2), 95-117.

Martel, C. (2013). Fiche réflexive TPaCK : Mise à l'essai et validation. Manuscrit non publié, Université de Sherbrooke, Performa, Sherbrooke.

Moroney, M. et Haigh, M. (2011). A lens on educational technology professional development opportunities: development of a general purpose Technological, Pedagogical et Content Knowledge questionnaire. Journal of Applied Research in Education, 15(1-2), 1-16.

Poellhuber, B. et Fournier-St-Laurent, S. (2014). Les TIC pour favoriser et soutenir l'apprentissage. In L. Menard et L. St-Pierre (dir.), Se former à la pédagogie de l'enseignement supérieur (p. 157-208). Montréal : les éditions AQPC.

Raby, C. et Viola, S. (2007). Courants pédagogiques, objets et agents d'apprentissage. In C. Raby et S. Viola (dir.), Modèle d'enseignement et théories d'apprentissage : de la pratique à la théorie (p. 18-36). Anjou : les éditions CEC.

Simmoneaux, J. (2011). Quelles postures épistémologiques pour une éducation au développement durable? Colloque international francophone. Le développement durable : débats et controverses, Clermont-Ferrand (France) : Université Blaise Pascal. Site téléaccessible à l'adresse : http:// www.oeconomia.net/private/colloqueddiufm/39.colloquedd-simmoneaux.pdf

\section{NOTES}

1. Le 2e cycle universitaire correspond au master français. 
2. Nous avons ainsi traduit l'intersection des trois connaissances (Technologiques, Pédagogiques et Disciplinaires) que propose l'auteur. Voir les explications à l'adresse: http:// performatic.espaceweb.usherbrooke.ca/?page_id=197

3. Le réseau collégial québécois bénéficie d'organismes et partenaires dont la mission est justement de développer ces ressources tels que le Centre collégial de développement du matériel didactique (CCDMD) : http://www.ccdmd.qc.ca/fr/repertoire/ ; l'Association pour les applications pédagogiques de l'ordinateur au post-secondaire (APOP) : https://apop.qc.ca/ ; Le projet Eurêka http://eureka.ntic.org/ ; le carrefour des ressources pédagogiques numériques en enseignement collégial http://www.profweb.ca/ ; le carrefour des jeux virtuels http:// www.savie.qc.ca/CVJE2/

\section{RÉSUMÉS}

À l'heure où la pertinence de l'intégration des TIC en éducation n'est plus à démontrer, la difficulté de faire valoir ces technologies auprès du personnel enseignant demeure un constat fréquent. Cette réticence à les inclure dans l'axe enseignement-apprentissage prend différentes formes, la première étant l'impression d'une inefficacité pédagogique. En effet, malgré l'existence d'outils technopédagogiques novateurs, ces derniers sont encore peu utilisés, l'enseignement magistral demeurant encore la méthode privilégiée. Or, innover signifie être en rupture avec des habitudes, avec des modèles bien ancrés et éprouvés. Par conséquent, comment favoriser cette rupture avec l'approche traditionnelle dominante auprès des enseignantes et enseignants ? L'expérimentation que nous avons réalisée auprès de trois groupes de participants composés d'enseignants et de conseillers pédagogiques de l'ordre collégial* dont cet article rend compte, nous permet de proposer une façon d'y arriver en stimulant un questionnement intégrant les dimensions liées aux connaissances disciplinaires et à la pédagogie, à l'instar du cadre de référence appelé le TPaCK (Mishra et Koehler, 2006). Les collectes de données se sont déroulées en juin 2013 et en février 2014. Grâce à cet exercice, un répertoire de ressources numériques technopédagogiques a non seulement été créé, mais la pertinence d'un outil réflexif favorisant l'identification et l'intégration des technologies dans l'enseignement a également été évaluée. L'analyse des scénarios identifiés a permis de tirer quelques constats et recommandations permettant d'optimiser l'utilisation du TPaCK.

* L'ordre collégial au Québec fait partie, au même titre que l'ordre universitaire, de l'enseignement supérieur et correspond à la dernière ou aux deux années de lycée français et à une année préuniversitaire.

At a time when the relevance of ICT integration in education is well established, there remains the difficulty of promoting such technologies among teachers. This reluctance on the teachers' part to include these technologies in the teaching-learning continuum takes various forms, beginning with their impression of its being an ineffective pedagogy. Indeed, despite the existence of innovative pedagogical tools promoting digital education, these tools are still little used, and lectures remain the preferred method of instruction. Innovation means breaking with habits and with well-entrenched, proven models. Consequently, how can this change be promoted among teachers? The experimentation we carried out with three groups of participants composed of college-level teachers and pedagogical counsellors [1] on which this article reports, enables us to propose a solution by integrating into their questioning the didactic and pedagogical dimensions, as in the TPACK framework (Mishra and Koehler, 2006). Data 
collection took place in June 2013 and February 2014.This exercise not only helped create a directory of techno-pedagogical resources, it also allowed for the evaluation of a reflective tool that promotes the identification and integration of technologies in teaching. The analysis of the resources identified gave rise to some observations and recommendations for optimizing the use of TPACK.

INDEX

Mots-clés : fiche réflexive TPaCK, intégration des technologies en classe au collégial, questionnement technopédagogique

Keywords : integrating Technologies in the College Classroom, techno-pedagogical reflective tool, TPaCK reflexive worksheet

\section{AUTEURS}

\section{CHRISTINE GAUCHER}

Conseillère pédagogique, Secteur PERFORMA de l'Université de Sherbrooke

\section{CLAUDE MARTEL}

Conseiller pédagogique TIC, Secteur PERFORMA de l'Université de Sherbrooke

\section{LÉANE ARSENAULT}

Directrice (2006-2014), Secteur PERFORMA de l'Université de Sherbrooke 